Draft VERsion July 26, 2021

Preprint typeset using $\mathrm{LAT}_{\mathrm{E}} \mathrm{X}$ style emulateapj v. 08/22/09

\title{
ON THE DISTANCE OF THE MAGELLANIC CLOUDS USING CEPHEID NIR AND OPTICAL-NIR PERIOD-WESENHEIT RELATIONS
}

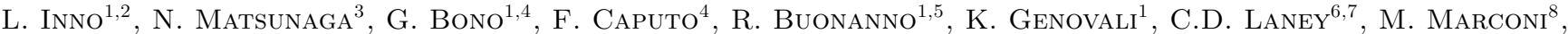 \\ A.M. Piersimoni ${ }^{5}$, F. Primas ${ }^{2}$, M. Romaniello ${ }^{2}$ \\ (Dated: drafted July 26, 2021 / Received / Accepted) \\ Draft version July 26, 2021
}

We present the largest near-infrared (NIR) data sets, $J H K_{\mathrm{S}}$, ever collected for classical Cepheids in the Magellanic Clouds (MCs). We selected fundamental (FU) and first overtone (FO) pulsators, and found 4150 (2571 FU, 1579 FO) Cepheids for Small Magellanic Cloud (SMC) and 3042 (1840 FU, 1202 FO) for Large Magellanic Cloud (LMC). Current sample is 2-3 times larger than any sample used in previous investigations with NIR photometry. We also discuss optical VI photometry from OGLE-III. NIR and optical-NIR Period-Wesenheit (PW) relations are linear over the entire period range $\left(0.0<\log P_{\mathrm{FU}} \leq 1.65\right)$ and their slopes are, within the intrinsic dispersions, common between the MCs. These are consistent with recent results from pulsation models and observations suggesting that the PW relations are minimally affected by the metal content. The new FU and FO PW relations were calibrated using a sample of Galactic Cepheids with distances based on trigonometric parallaxes and Cepheid pulsation models. By using FU Cepheids we found a true distance moduli of $18.45 \pm 0.02$ (random) \pm 0.10 (systematic) mag (LMC) and $18.93 \pm 0.02$ (random) \pm 0.10 (systematic) mag (SMC). These estimates are the weighted mean over $10 \mathrm{PW}$ relations and the systematic errors account for uncertainties in the zero-point and in the reddening law. We found similar distances using FO Cepheids $(18.60 \pm 0.03$ (random) \pm 0.10 (systematic) mag [LMC] and $19.12 \pm 0.03($ random $) \pm$ 0.10 (systematic) mag $[\mathrm{SMC}]$ ). These new MC distances lead to the relative distance, $\Delta \mu=0.48 \pm$ $0.03 \mathrm{mag}(\mathrm{FU}, \log P=1)$ and $\Delta \mu=0.52 \pm 0.03 \mathrm{mag}(\mathrm{FO}, \log P=0.5)$, which agrees quite well with previous estimates based on robust distance indicators.

Subject headings: Magellanic Clouds — stars: variables: Cepheids — stars: distances — stars: oscillations

\section{INTRODUCTION}

Recent detailed investigations indicate that $2 \%-3 \%$ of the systematic error affecting the Hubble constant estimate is due to the Cepheid distance to the Large Magellanic Cloud (LMC Freedman \& Madore 2010a; Riess et al. 2011, Freedman et al. 2012). Moreover, the Magellanic Clouds (MCs) are fundamental benchmarks to constrain the accuracy and the precision of the most popular primary distance indicators (Pietrzyński et al. 2010; Matsunaga et al. 2009, 2011). The decrease of a factor two in metallicity between the LMC and the Small Magellanic Cloud (SMC) makes these galaxies also excellent laboratories to constrain the possible dependence of different standard candles on the metal content. Although, the

\footnotetext{
${ }^{1}$ Dipartimento di Fisica, Università di Roma Tor Vergata, via della Ricerca Scientifica 1, I-00133 Rome, Italy; laura.inno@roma2.infn.it

2 European Southern Observatory, Karl-Schwarzschild-Str. 2,D-85748 Garching bei Munchen, Germany

${ }^{3}$ Kiso Observatory, Institute of Astronomy, School of Science, The University of Tokyo 10762-30, Mitake, Kiso-machi, Kiso-gun,3 Nagano 97-0101, Japan

${ }^{4}$ INAF-OAR, via Frascati 33, I-00040 Monte Porzio Catone, Rome, Italy

${ }^{5}$ INAF-Osservatorio Astronomico di Collurania, via M. Maggini,I-64100 Teramo, Italy

${ }_{6}^{6}$ Department of Physics and Astronomy, N283 ESC, Brigham Young University, Provo, UT 84601, USA

${ }^{7}$ South African Astronomical Observatory, P.O. Box 9, Observatory 7935 , South Africa

8 INAF-Osservatorio Astronomico di Capodimonte, via Moiarello 16,I-80131 Napoli, Italy
}

MC Cepheids play a crucial role in many astrophysical problems, the number of homogeneous optical $(B, V, R, I)$ and near-infrared (NIR; $\left.J, H, K_{\mathrm{S}}\right)$ data sets is quite limited.

The most extensive surveys in the optical bands $(V, I)$ were performed by micro-lensing experiments (MACHO, EROS, OGLE). The MACHO project collected $R, I$ band data for $~ 1900$ Cepheids in the LMC (Skrutskie et al. 2006, Alcock et al. 2000, Welch et al. 1999), while EROS collected $V, R$ band data for $\sim 300$ and $\sim 600$ Cepheids in the $\mathrm{LMC}$ and in the $\mathrm{SMC}$, respectively (Beaulieu \& Marquette 2000). The most complete sample of MC Cepheids was collected by OGLE-III (Soszyński et al. 2008, 2010). Their catalog includes $V, I$ band light curves for more 7000 Cepheids (LMC: 2000 fundamental [FU], 1000 first overtone [FO]; SMC: 2500 $\mathrm{FU}, 1500 \mathrm{FO})$. Accurate distance determinations to the MCs based on optical Period-Wesenheit (PW) relations have also been provided by Udalski et al. (1999), Bono et al. (2002), Groenewegen \& Salaris (2003) and Ngeow et al. (2009). More recently, Di Criscienzo et al. (2012) provided a detailed theoretical investigation concerning the PW relations in the Sloan Digital Sky Survey bands.

The NIR data bases for MC Cepheids are significantly smaller: Laney \& Stobie (1986, 1994) collected NIR light curves for $44 \mathrm{MC}$ Cepheids (21 LMC, 23 SMC), while Welch et al. (1987) for 91 SMC Cepheids. More recently Persson et al. (2004, hereinafter P04) collected NIR light curves for 92 LMC Cepheids. More recently, accurate $K$ band photometry (12 phase points per variable) was 
collected by Ripepi et al. (2012, hereinafter R12) in two LMC fields located around 30 Doradus (172 FU, 152 FO) and the South Elliptical Pole (11 Cepheids). They provided, by using also literature data, accurate estimates of Period-Luminosity (PL), PW, and Period-LuminosityColor relations for both FU and FO Cepheids. One of the key advantages in using NIR data is that the pulsation amplitude decreases for increasing wavelength and the estimate of the mean magnitude becomes easier. The previous largest set of single epoch measurements for $\mathrm{MC}$ Cepheids was collected by Groenewegen (2000) (LMC: 713 FU, 450 FO; SMC: 1200 FU, 675 FO) using 2MASS and DENIS data sets. The same approach was also followed by Nikolaev et al. (2004) using MACHO and 2MASS data sets (LMC: 1357 FU, 749 FO) and more recently by Ngeow et al. (2009) using the 2MASS data set (LMC: $1761 \mathrm{FU}$ ) and the mid-infrared SAGE Spitzer (Meixner et al. 2006) data set (LMC: 1759 FU).

In this investigation, we provide new MC distances using a new sample of single-epoch $J, H, K_{\mathrm{S}}$ measurements of a significant fraction of MC Cepheids $(\sim 80 \%)$ detected by OGLE. In particular, in Section 2 we discuss the NIR and optical data sets we adopted in this investigation together with the criteria to select both FU and FO Cepheids. In Section 3 we present the PW relations, while in Section 3.1 we focus our attention on the linearity and the metallicity dependence of NIR and opticalNIR PW relations. Empirical and theoretical absolute calibrations of the PW relations are addressed in Section 3.2. The summary of the results and more detailed discussion concerning pros and cons of the two independent calibrations are given in Section 4, while in Section 5 we briefly outline some possible future avenues concerning the developments of this project.

\section{DATA SETS AND DATA SELECTION}

The Cepheid intrinsic parameters are taken from the OGLE-III catalog (Soszyński et al. 2008, 2010). We adopted the following Cepheid parameters: pulsation period, position (right ascension and declination), mean $V$ and $I$ band magnitude, $I$ band amplitude, epoch of maximum and pulsation mode. The optical OGLEIII Cepheid catalog was cross-correlated with the NIR catalog of the IRSF/SIRIUS Near-Infrared Magellanic Clouds Survey provided by Kato et al. (2007). The single-epoch $J, H, K_{\mathrm{S}}$ magnitudes for the OGLE-III FU Cepheids were extracted by Matsunaga et al. (2011). In this investigation we also included FO Cepheids. We ended up with a sample of 3042 LMC (1840 FU, 1202 FO) and 4150 SMC (2571 FU, 1579 FO) Cepheids with three NIR $\left(J, H, K_{\mathrm{S}}\right)$ single-epoch measurements. The IRSF/SIRIUS $J, H, K_{\mathrm{S}}$ measurements were transformed into the 2MASS NIR photometric system following Kato et al. (2007).

The mean magnitudes of FU Cepheids were estimated using the NIR template light curves provided by Soszyński et al. (2005). To assess the accuracy of this method, we compared our estimates of mean magnitudes with the mean magnitudes for LMC Cepheids based on finely sampled light curves (P04). The template light curves and the mean magnitude by P04 were also transformed into the 2MASS NIR photometric system following Carpenter (2001). Figure 1 shows that the intrinsic dispersion decreases by a factor of two when moving from the single-epoch measurements to the mean magnitudes based on the template ( 0.12 versus $0.05 \mathrm{mag})$. The total error budget of the mean magnitudes estimated using the template light curve is given by $\sigma_{\lambda_{i}}^{2}=\sigma_{m_{i}}^{2}+\sigma_{\text {cal }}^{2}+\sigma_{\text {rph }}^{2}$, where $\sigma_{m_{i}}^{2}$ is the intrinsic photometric error, with a typical value of $\sim 0.03$ at $16 \mathrm{mag}$ in $J, H, K_{\mathrm{S}} ; \sigma_{\text {cal }}^{2}$ is the error due to the transformation into the 2MASS photometric system, it is of the order of $0.01 \mathrm{mag}$ for UKIRT, LCO and IRSF systems; $\sigma_{\text {rph }}^{2}$ is the scatter due to the random phase sampling, it is given by the template algorithm and it is $\sim 0.05 \mathrm{mag}$ (Soszyński et al. 2005).

For the FO Cepheids the mean magnitude is based on the single epoch measurements, since template light curves are not available for these pulsators. It is worth mentioning, that the mean magnitudes of these pulsators are less affected by their random sampling, since their luminosity amplitude is on average three times smaller than for FU Cepheids (Freedman \& Madore 2010b). The errors of the FO mean magnitudes were estimated using the above relation, but the term $\sigma_{\mathrm{rph}}^{2}$ gives the typical semi-amplitude of FO light curves $(\sim 0.10 \mathrm{mag})$. We plan to address the discussion concerning the template light curve for FO Cepheids and their errors in a forthcoming paper.

\section{PERIOD WESENHEIT RELATIONS}

The Wesenheit indices, introduced by Madore (1982), are pseudo-magnitudes closely related to apparent magnitudes, but minimally affected by uncertainties on reddening. On the basis of two magnitudes, $m_{\lambda_{1}}$ and $m_{\lambda_{2}}$, we can define a Wesenheit index:

$$
W\left(\lambda_{2}, \lambda_{1}\right)=m_{\lambda_{1}}-\left[\frac{A\left(\lambda_{1}\right)}{E\left(m_{\lambda_{2}}-m_{\lambda_{1}}\right)}\right] \times\left(m_{\lambda_{2}}-m_{\lambda_{1}}\right)
$$

where $\lambda_{1}>\lambda_{2}$ and $\frac{A\left(\lambda_{1}\right)}{E\left(m_{\lambda_{2}}-m_{\lambda_{1}}\right)}$ is the total to selective extinction for the given filters $-\left\{\lambda_{i}=V, I, J, H, K_{\mathrm{S}}\right\}-$ and for the adopted reddening law. The clear advantage in using the Wesenheit indices is that they are minimally affected by uncertainties affecting reddening corrections for Galactic and extragalactic Cepheids. Once we fix the reddening law (Cardelli et al. 1989) and we assume $R_{V}=\frac{A(V)}{A(B)-A(V)}=3.23$, we obtain the following selective absorption ratios, namely $A_{I} / A_{V}=0.61 ; A_{J} / A_{V}=0.29$; $A_{H} / A_{V}=0.18 ; A_{K_{S}} / A_{V}=0.12 \mathrm{mag}$.

By combining the five optical-NIR $\left(V I J H K_{\mathrm{S}}\right)$ mean magnitudes, we can compute 10 Wesenheit indices for each Cepheid in the sample, and in turn, $10 \mathrm{PW}$ relations of the form $W\left(\lambda_{2}, \lambda_{1}\right)=a+b \times \log P$, where $\mathrm{P}$ is the pulsation period in days. We decided to analyze separately FU and FO Cepheids to overcome possible systematic uncertainties that the 'fundamentalization' of the FO periods might introduce in the estimate of the PL relations (Feast \& Catchpole 1997, Marengo et al. 2010). Therefore, we computed independent PW relations for FU and FO Cepheids. We performed a linear fit of the data to identify possible outliers. We have included data up to $4 \sigma$ from the central location by using the robust Biweight location estimator (Fabrizio et al. 2011). We ended up with a sample of $\sim 4000$ SMC $(\sim 2500, \mathrm{FU}$; $\sim 1500$, FO $)$ and $\sim 3000$ LMC $(\sim 1800$, FU; $\sim 1100$, FO $)$ Cepheids. For approximately three dozen Cepheids with 
period $\gtrsim 20$ days the $I$ band is saturated in the OGLEIII data-set, and therefore we cannot apply the template. The NIR mean magnitudes for these Cepheids were taken from P04 and transformed into the 2MASS photometric system (see green dots in Figure 2).

We then performed a linear regression of the NIR data and the results for the three PW relations are showed in Figure 2 (see also Table 1). From top to bottom each panel shows FU (red and green dots) and FO (blue dots) LMC (left) and SMC (right) Cepheids. The PW relations are over-plotted as black lines. Data plotted in Figure 2 display four relevant findings concerning the NIR PW relations. (1)The FU and the FO PW relations are linear over the entire period range. (2)The intrinsic dispersion of the SMC PW relations are a factor of two larger than for the LMC PW relations. The difference is mainly caused by depth effects in the former system (van den Bergh 2000). (3)For each PW relation the difference in the slope between LMC and SMC Cepheids is small. Data listed in Tables 1 and 2 indicate that it is, on average, smaller than $0.8 \sigma_{t o t}$, where $\sigma_{t o t}$ is the sum in quadrature of the dispersion of LMC and SMC PW relations.

This indicates a minimal dependence of the NIR PW relations on the metal content. (4)The width in temperature of the FO instability strip is narrower than the instability strip for FU Cepheids (Bono et al. 2000), but the intrinsic dispersions are not significantly different. The lack of a template light curve for FO Cepheids increases the dispersion of their mean magnitudes.

The above findings support recent theoretical (Bono et al. 2010) and empirical (Majaess et al. 2011) investigations. The main advantage of the current approach is that the results rely on NIR single epoch measurements that are $2-3$ times larger than any previous investigation (Groenewegen 2000). In order to constrain the possible occurrence of systematic errors in the NIR PW relations, we also computed the optical-NIR PW relations using the $V, I$ mean magnitudes provided by OGLE-III. Figures 3 and 4 show the optical-NIR PW relations for FU (red dots) and FO (blue dots) LMC and SMC Cepheids, respectively. Once again, we found that the $\mathrm{PW}$ relations are linear and the slopes are minimally affected by the difference in metal content (see Table 1). Current results concerning the linearity of NIR and optical-NIR PW relations support previous findings by Ngeow et al. (2005) and Madore \& Freedman (2009).

\subsection{Linearity of $P W$ Relations}

To constrain on a more quantitative basis the linearity of NIR PW relations we estimated the distance of individual Cepheids from the least squared solution. The residuals for $\mathrm{FU}$ Cepheids plotted in the top panels of Figure 5 do not show any trend as a function of the pulsation period. To further constrain this evidence, we performed a linear fit to the residuals and we found that the zero-points, the slopes and the means attain vanishing values. Moreover, the dispersions are typically smaller than $0.2 \mathrm{mag}$. The same outcome applies to the FO Cepheids (see bottom panels of Figure 5). Note that the residuals of FO PW relations are larger than the residuals of the FU PW relations, since for the former ones we lack template light curves. The residuals referred to SMC are larger than the residuals of $\mathrm{LMC}$ due to depth effects. The anonymous referee explicitly asked a quantitative analysis concerning the linearity of the PW relations for both FU and FO SMC Cepheids. To our knowledge there is no clear physical reason why FU and FO NIR PW relations should show a break, therefore, we decided to constrain the possible occurrence using different breaks in period. We split the entire Cepheid sample by adopting a break in period at $\log P=0.45$. This means that we assume as short-period Cepheids those with $\log P \leq 0.45$, while the long-period ones are those with $0.45<\log P \leq 1.65$ (FU) and with $0.45<\log P \leq 0.65$ (FO). The zero-points and the slopes for FU NIR PW relations listed in Table 3 show that their errors are a factor of 3-4 larger than the errors of the linear regressions based on the entire sample. This trend is expected, since the number of Cepheids included in the two new linear regressions decreases from a factor of three (long-period) to $50 \%$ (short-period). On the other hand, the dispersions of the new PW relations are either similar (short-period) or on average smaller (longperiod). The new FO PW relations show similar trends concerning the intrinsic errors on the zero-points, on the slopes and on the dispersions.

The break in period is defined somewhat arbitrarily, therefore, we decided to perform the same test, but using a break at $\log P=0.40$ and $\log P=0.35$. Current empirical evidence suggests that optical PL relations of SMC Cepheids show a break in period at $\log P \approx 0.4$ (Sandage et al. 2009), while for LMC Cepheids the break seems to be at $\log P \approx 1$ (Sandage et al. 2004). The results concerning the new NIR PW relations are listed in Table 3 and indicate that the short-period $\mathrm{PW}$ relations are quite similar to the global PW relations, i.e., the PW relations covering the entire period range. This trend is -once again- expected, since more than $2 / 3$ of the Cepheid sample is in the short-period range. The evidence that linear regressions with an arbitrary break in period, give PW relations with either similar or marginally smaller dispersion is also expected. This is the consequence of the increase in the degrees of freedom of the linear regressions. However, this does not mean that the PW relations with a break in period are a better representation of observations. To address this issue on a more quantitative basis, we devised a new empirical test based on the relative distance between SMC and LMC. The MC relative distance is quite solid, since different standard candles provide similar estimates.

By adopting both NIR and optical-NIR PW relations, we found that the relative distance modulus based on $\mathrm{FU}$ Cepheids and at $\log P=1$ is $\Delta \mu=0.48 \pm 0.03$ mag. This evaluation agrees quite well with similar estimates available in the literature (see Section 4). To further constrain the intrinsic accuracy of the NIR PW relations with a break in period, we computed three new $\mathrm{PW}\left(J, K_{\mathrm{S}}\right)$ relations for LMC Cepheids. Following Sandage et al. (2004), we adopted a break in period at $\log P=1$. 'The zeropoints and the slopes of the new NIR PW relations are listed in Table 4 . We estimated the MC relative distances by using the short-period and the long-period PW relations. The relative distances based on the former ones were estimated at $\log P=0.3$, while those based on the latter ones were estimated at $\log P=1.0$. The results listed in Table 5 indicate -as expected- that the $\mathrm{MC}$ relative distances based on short-period $\mathrm{PW}$ rela- 
tions agree quite well with the MC relative distances based on global PW relations. The main difference is that the relative distance based on short-period PW relations have intrinsic errors, estimated by propagating the errors on both the coefficients and the dispersion of the individual PW relations, that are on average a factor of two larger than those ones based on the global PW relations. The same outcome applies to the MC relative distances based on the long-period PW relations. However, their intrinsic errors are larger and they also show a larger spread among the three different NIR PW relations. Note that the $\mathrm{MC}$ relative distance based on the long-period $\mathrm{PW}(J, H)$ relations are systematically smaller than the others, because the zero-point of the long-period PW relation for LMC Cepheids is larger than the zero-point of the global PW relation (15.949 versus 15.876).

We repeated the same test by using two different pivot periods, namely $\log P=0.2$ for the short-period and $\log P=1.2$ for the long-period PW relations and the results are quite similar. We also performed the same test using NIR FO PW relations and the outcome is -once again- quite similar. Note that the intrinsic errors on the coefficients of the long-period FO PW relations are larger than the errors of the short-period ones, since the Cepheid sample in the former period interval is from a factor of five to a factor of 10 smaller than in the latter one.

The above findings indicate that the PW relations with arbitrary breaks in period when compared with global PW relations have larger intrinsic errors on the coefficients of the linear regressions and roughly equivalent dispersions.

However, the MC relative distances based on the former ones are characterized by intrinsic errors that are, on average, a factor of two larger than the latter ones. Thus further supporting the use of global NIR PW relations.

This provides an independent support to the results concerning the linearity of both optical and NIR PW relations for FU Cepheids by Persson et al. (2004); Bono et al. (2010); Ngeow (2012, and references therein). We found that optical and NIR PW relations for FO Cepheids are also linear over the entire period range, supporting previous findings by Ngeow et al. (2005) and Madore \& Freedman (2009). We are thus facing the empirical evidence that optical and NIR PL relations for FU Cepheids do show a change in the slope for $\log P \approx 0.4$ (Sasselov et al. 1997; Bono et al. 1999, Ngeow et al. 2005; Koen et al. 2007; Matsunaga et al. 2011). 'The difference between the PL and the PW relations is mainly due to the fact that the latter is mimicking, as originally suggested by Bono \& Marconi (1999), a PLC relation.

\subsection{Metallicity dependence of the PW relations}

To further constrain the metallicity dependence of the NIR PW relations, we performed a detailed comparison with similar estimates available in the literature. The middle panel of Figure 6 shows the difference between the slope of the $\mathrm{PW}\left(J, K_{\mathrm{S}}\right)$ relations we estimated for LMC (black line) and SMC (green line) Cepheids with similar PW relations for Galactic Cepheids (see Table 6) derived by Storm et al. (2011a, hereinafter S11a; red line) and by Ngeow (2012, hereinafter N12; purple line). The standard deviations plotted in the same figure clearly indicate that current Magellanic and Galactic NIR PW relations do agree within $1 \sigma$. The difference in the slope between our SMC and Galactic $\mathrm{PW}\left(J, K_{\mathrm{S}}\right)$ relations is, on average, smaller than $0.3 \sigma(\mathrm{N} 12)$ and $0.4 \sigma$ (S11a).

The anonymous referee suggested to perform the same comparison for the optical $\mathrm{PW}(V, I)$ relation. The top panel of Figure 6 shows the difference between the slope of the $\mathrm{PW}(V, I)$ relations we estimated for LMC (black line) and SMC (green line) Cepheids with similar PW relations for Galactic Cepheids (see Table 6) derived by S11a (red line) and Benedict et al. (2007, hereinafter B07; blue line). The standard deviations plotted in the same figure clearly indicate that current Magellanic and Galactic optical PW relations do agree within $1 \sigma$. The difference in the slope of the $\mathrm{PW}(V, I)$ relation between our metal-poor stellar system $(\mathrm{SMC},[\mathrm{Fe} / \mathrm{H}]=-0.75)$ and our metal-rich stellar system (Galaxy, $[\mathrm{Fe} / \mathrm{H}]=-0.18$ to $+0.25)$ is, on average, smaller than $\sim 0.1 \sigma$ (B07) and $\sim 0.9 \sigma(\mathrm{S} 11 \mathrm{a})$. The bottom panel of Figure 6 shows the difference between the slope of the $\mathrm{PW}\left(V, K_{\mathrm{S}}\right)$ relations we estimated for LMC (black line) and SMC (green line) Cepheids with the PW relation for LMC Cepheids (see Table 6) derived by R12 (gray line). Data plotted in this figure clearly indicate the good agreement between the two independent LMC slopes. Moreover, current SMC and LMC PW relations do agree within $1 \sigma$. The other NIR and optical-NIR PW relations provide similar results. The quoted numbers indicate that the $\mathrm{PW}$ relations are, in the metallicity range covered by Magellanic Cepheids, independent of metal abundance. The extension into the more metal-rich regime does require more accurate measurements for Galactic Cepheids.

\subsection{Absolute calibration of the $P W$ relations}

To estimate the distances to the MCs, we combined our new comprehensive sets of PW relations with recent findings concerning absolute magnitudes of Galactic Cepheids. We followed the same approach suggested by P04 to calibrate the LMC PW relations and adopted the 10 FU Galactic Cepheids with Hubble Space Telescope trigonometric parallaxes (Benedict et al. 2007). To calibrate the FO PW relations, we adopted the $H$ ipparcos trigonometric parallaxes for Polaris provided by van Leeuwen et al. (2007). The mean $J, H, K_{\mathrm{S}}$ magnitudes for the calibrating Galactic Cepheids are from Laney \& Stobie (1992). We estimated the true distance modulus $-\mu$ of both LMC and SMC by using the quoted calibrators and by imposing the slope of individual PW relations for FU and FO Cepheids (see Column 6 of Table 1).

Note that the true distance moduli for FU Cepheids were estimated as the weighted mean of the $\mu_{i}$ of individual calibrating Cepheids. The associated error on $\mu$ is the sum in quadrature of the weighted error on the distance and of the intrinsic dispersion associated with the linear regression (see Column 5 of Table 1). The weighted means based on the FU PW relations give $\mu(\mathrm{LMC})=18.45 \pm 0.02$ and $\mu(\mathrm{SMC})=18.93 \pm 0.02 \mathrm{mag}$, while the weighted means based on FO PW relations give $\mu(\mathrm{LMC})=18.60 \pm 0.03$ and $\mu(\mathrm{SMC})=19.12 \pm 0.03 \mathrm{mag}$.

To constrain the possible occurrence of deceptive errors in the absolute zero-point, we performed an independent zero-point calibration using predicted FU PW relations for Magellanic Cepheids provided by Bono et al. (1999) and Marconi et al. (2005). Recent investigations indicate 
that theory and observations agree quite well concerning optical and optical-NIR PW relations (Bono et al. 2010). The true distance moduli based on the new zero-point calibration are listed in column seven of Table 1 . The error associated to individual distance moduli is the standard deviation from the theoretical PW relation. The new weighted means based on the FU PW relations give $\mu(\mathrm{LMC})=18.56 \pm 0.02$ and $\mu(\mathrm{SMC})=18.93 \pm 0.02 \mathrm{mag}$. Interestingly enough, the two independent calibrations for FU Cepheids do provide weighted true distance moduli to the MCs that agree quite well (DM $\lesssim 0.11 \mathrm{mag})$. This finding appears even more compelling if we take into account that we are using independent NIR and optical data sets together with independent theoretical and empirical calibrators.

Current zero-point calibration for FO PW relations relies on the trigonometric parallax of a single object (Polaris, van Leeuwen et al. 2007). Absolute distances for FO Galactic and Magellanic Cepheids based on the IRSB method are not available. To overcome this problem, we decided to use predicted FO PW relations for Magellanic Cepheids provided by Bono et al. (1999) and Marconi et al. (2005). The true distance moduli based on the new zero-point calibration are listed in Column 7 of Table 1. The error associated to individual distance moduli are the dispersions from the theoretical PW relation. The new weighted means based on the FO PW relations give $\mu(\mathrm{LMC})=18.51 \pm 0.02$ and $\mu(\mathrm{SMC})=19.02 \pm 0.02 \mathrm{mag}$. The two independent empirical calibrations for $\mathrm{FU}$ and $\mathrm{FO}$ Cepheids provide weighted true distance moduli to the MCs that differ from 0.15 (LMC) to 0.19 (SMC) mag. On the other hand, the weighted true distance moduli based on the theoretical calibrations differ at the level of a few hundredths of mag. The difference between the two empirical calibrations is due to the fact that the empirical calibrations for FO PW relations rely on a single object (see Section 4).

However, data listed in Table 1 indicate that the $\mathrm{PW}(J, H)$ and the $\mathrm{PW}(I, H)$ relations for $\mathrm{FU}$ and $\mathrm{FO}$ Cepheids, calibrated using the Galactic Cepheids with trigonometric distances, provide true distance moduli that differ at the $2 \sigma-3 \sigma$ level from the weighted mean. The evidence that distances based on PW relations, calibrated using theoretical predictions for MC Cepheids (L. Inno et al. 2013, in preparation), show smaller differences indicates that the main culprit seems to be the precision of the $H$ band zero-point calibration. However, the difference in the weighted mean distances, provided by the two independent zero-point calibrations for FU Cepheids, is smaller than $5 \%$ (LMC: $49.0 \pm 1.2$ versus $51.5 \pm 1.2 \mathrm{kpc}$; SMC: $61.1 \pm 2.2$ versus $68.8 \pm 2.3 \mathrm{kpc})$. Moreover, the total uncertainty of current LMC and SMC distances is at the $\sim 2 \%$ and at the $\sim 4 \%$ level, respectively. Note that we obtain very similar distances if we neglect the distances based on the $\mathrm{PW}(J, H)$ and $\mathrm{PW}(I, H)$ relations, namely $18.47 \pm 0.02$ (trigonometric parallaxes) and $18.57 \pm 0.03$ (theory) mag.

To further constrain the possible sources of systematic errors in current distance estimates, we also constrained the impact of the adopted reddening law. In a recent investigation Kudritzki \& Urbaneja (2012) suggested that distance determinations based on the $\mathrm{PW}$ relations may be affected by changes in the reddening law either in the Galaxy or in external stellar systems. To constrain this effect we computed a new set of PW relations by adopting the reddening law by McCall (2004). We found that the difference in the true distance moduli, based on the two different reddening laws, is on average $\sim 0.01$ mag. The mild dependence of current distance determinations on the reddening law might also be due to the fact that the selective absorption ratios of optical-NIR PW relations are less sensitive to the fine structure of the reddening law. The selective absorption ratios given in Section 3 indicate that the coefficient of the color term of the $\mathrm{PW}(V, K)$ relation is at least one order of magnitude smaller than the coefficients of $\mathrm{PW}(J, H)$ and $\mathrm{PW}\left(H, K_{\mathrm{S}}\right)$ relations (0.13 versus 1.63 and $\left.1.92 \mathrm{mag}\right)$. This evidence indicates that the difference in the true distance moduli based on $\mathrm{PW}(J, H)$ and $\mathrm{PW}\left(H, K_{\mathrm{S}}\right)$ relations might also be caused either by photometric error in the mean magnitudes or by changes in the reddening law along the line-of-sight of the HST Galactic calibrating Cepheids.

\section{SUMMARY AND DISCUSSION}

We present new true distance modulus determinations of the MCs using NIR and optical-NIR PW relations. The NIR PW relations were estimated adopting the largest data set of $J, H, K_{\mathrm{S}}$ single epoch measurements ever collected for MC Cepheids. The optical $V, I$ measurements come from the OGLE-III data set. We ended up with a sample of 4150 (2571, FU; 1579, FO; SMC) and 3042 (1840, FU; 1202, FO; LMC) Cepheids. We estimated independent $\mathrm{PW}$ relations for both $\mathrm{FU}$ and $\mathrm{FO}$ Cepheids. The slopes of the current FU PW relations agree quite well with similar estimates available in the literature. We found that they agree at $1.2 \sigma$ level with the slopes of the NIR PW relations for LMC Cepheids derived by $\mathrm{P} 04$. The agreement is even better if we compare our slopes for the $\mathrm{PW}\left(J, K_{\mathrm{S}}\right)$ relations with the slopes recently provided by Storm et al. (2011b, hereinafter S11b) for the LMC (LMC: $-3.31 \pm 0.09$ versus $3.365 \pm 0.008)$. The above findings are even more relevant if we take into account that current slopes are based on data samples that are from $80(\mathrm{~S} 11 \mathrm{~b})-30$ (P04) times to $\sim 3$ times (Groenewegen 2000) larger than the quoted samples. We cannot perform a similar comparison concerning the slopes of the FO PW relations, since to our knowledge they are not available in the literature.

Moreover, we found that both FU and FO PW relations are linear over the entire period range and their slopes attain, within the intrinsic dispersions, similar values in the MCs. The difference is, on average, smaller than $0.8 \sigma$. The difference between the slope of our SMC and Galactic $\mathrm{PW}\left(J, K_{\mathrm{S}}\right)$ relations available in the literature is, on average, smaller than $0.5 \sigma(0.3 \sigma, \mathrm{N} 12 ; 0.4 \sigma$, $\mathrm{S} 11 \mathrm{~b})$. The same outcome applies to optical bands, and indeed the difference in the slope between our SMC and Galactic $\mathrm{PW}(V, I)$ relations available in the literature is, on average, smaller than $\sim 0.1 \sigma$ (B07) and $\sim 0.9 \sigma$ (S11a). This supports the evidence for a marginal dependence of NIR and PW(V,I) relations on the metal content, as suggested by pulsation predictions and recent empirical investigations.

The new PW relations were calibrated using two independent sets of Galactic Cepheids with individual distances based either on trigonometric parallaxes or on theoretical models. By using FU Cepheids we found a true 
distance modulus to the LMC of $18.45 \pm 0.02$ (random) \pm 0.10 (systematic) mag and to the SMC of $18.93 \pm 0.02$ (random) \pm 0.10 (systematic) mag. These estimates are the weighted mean over the entire set of distance determinations. The random error was estimated by taking into account the intrinsic dispersion of individual PW relations. The systematic error is the sum in quadrature of the difference in $\mu$ introduced by the change in reddening law and in the zero-point calibration.

We found similar distances using FO Cepheids $18.60 \pm 0.03$ (random) \pm 0.10 (systematic) mag, LMC and $19.12 \pm 0.03$ (random) \pm 0.10 (systematic) mag, SMC. Once again the random errors were estimated by taking into account the intrinsic dispersion of individual PW relations, while the systematic ones are the sum in quadrature of the difference in $\mu$ introduced by the change in reddening law and in the zero-point calibration.

The two independent empirical calibrations for FU and FO Cepheids provide weighted true distance moduli to the MCs that differ for 0.15 (LMC) and 0.19 (SMC) mag. On the other hand, the weighted true distance moduli based on the theoretical calibrations differ at the level of a few hundredths of mag. The difference between the empirical and theoretical calibrations is due to the fact that the empirical calibrations for FO PW relations rely on a single object (see Section 3.2).

The relative distance of the MCs, for distance indicators minimally affected by the metal content, is independent of uncertainties affecting the zero-point calibration. We found that the weighted mean relative distance between SMC and LMC using FU Cepheids and the PW relation listed in Table $1(\log P=1)$ is $\Delta \mu=0.48 \pm 0.03$ mag. We applied the same approach by using FO Cepheids and we found $\Delta \mu=0.52 \pm 0.03 \mathrm{mag}(\log P=0.5)$. The errors on the weighted mean relative distances were estimated by using the dispersions of individual PW relations. The quoted determinations agree quite well with each other and with the recent estimate $\Delta \mu=0.47 \pm 0.15 \mathrm{mag}$ provided by S11b by using the IRSB method (see also Groenewegen 2000; Bono et al. 2010; Matsunaga et al. 2011).

The distance modulus we obtained for the LMC agrees quite well with the recent estimate provided by $\mathrm{S} 11 \mathrm{~b}$ (18.45 $\pm 0.04 \mathrm{mag})$ and by P04 (18.50 $\pm 0.05 \mathrm{mag})$ by using the NIR PL, PLC and PW relations. The difference is also minimal with the "classical" value $-18.50 \pm 0.10$ mag-(Freedman et al. 2001). The same conclusion can be reached if we compare the current estimate with recent distance moduli provided by Benedict et al. (2007. $18.50 \pm 0.03 ; H S T$ trigonometric parallaxes for Galactic Cepheids and the LMC slope of the optical PW relation); by Ngeow \& Kanbur (2008, 18.49 \pm 0.04 ; optical PL and PLC relations); by Freedman \& Madore (2010a $18.44 \pm 0.03$ (random) \pm 0.06 (systematic); PW $(V, I)$ relation for Galactic and LMC Cepheids ) and by Ngeow (2012, 18.531 $\pm 0.043 \mathrm{mag}$; NIR and optical-NIR PW relations) ${ }^{9}$. Moreover, our result also agrees with the most recent distance modulus - $18.477 \pm 0.033-$ provided by Scowcroft et al. (2011) and Freedman et al. (2012), using the Spitzer mid-IR band PL relations.

The distance modulus we obtained for the SMC is, once again, in very good agreement with the independent

9 Note that in the comparison of LMC distance moduli, we adopted the estimates that neglect the metallicity dependence. estimates provided by (Groenewegen 2000, 19.11 \pm 0.11 mag; Hipparcos trigonometric parallaxes and $\mathrm{PW}(V, I)$ relation) and $\mathrm{S} 11 \mathrm{~b}(18.92 \pm 0.14 \mathrm{mag})$.

\section{FINAL REMARKS}

The key feature of current findings is that the random errors associated to our distance determinations are very small, due to the fact that we adopt an homogeneous and accurate NIR data set and also because we are fully exploiting the use of NIR and optical-NIR PW relations. Moreover, the use of two independent zero-point calibrations and two different reddening laws indicate that the global uncertainty on the MC distances seems to be of the order of $1 \%$ by using either the 10 NIR/optical-NIR PW relations or the seven optical-NIR PW relations.

However, there are a few pending issues that need to be addressed in more detail in the near future.

1. The very good intrinsic accuracy of NIR and optical-NIR PW relations further support the findings by Bono et al. (2010, see their Figures 13 and 14) indicating that the difference between optical $(B, V)$ and NIR $\left(J, K_{\mathrm{S}}\right)$ PW relations can be adopted to constrain the metallicity correction(s) to the Cepheid distance scale based on optical PL relations. Moreover, current findings indicate that the error budget of the absolute distances based on PW relations is dominated by uncertainties in the zero-point. The solution of this problem appears quite promising in light of the fact that Gaia will be launched in approximately one year and the number of double eclipsing binaries including classical Cepheids is steadily increasing during the last few years (Pietrzyński et al. 2010, 2011). Moreover, new optical (OGLEIV Soszyński et al. 2012 ) and NIR (Galaxy:VVV, Minniti et al. 2010); (MCs:VMC, Cioni et al. 2011, R12) surveys will also provide new, homogenous and accurate mean magnitudes.

2. The above results provide an independent support to the plausibility of the physical assumptions adopted in current hydrodynamical models of variable stars. Indeed the distance moduli based on theoretical calibrations agree well with distance moduli based on empirical calibration. However, we still lack detailed investigations concerning the pulsation properties of Classical Cepheids in the metal-intermediate regime. In particular, we need a comprehensive analysis of the metallicity dependence of both PW relations and PeriodLuminosity-Color relations in the optical and in the NIR regime.

3. Accurate spectroscopic iron abundances are only available for roughly the $50 \%$ of Galactic Cepheids (Romaniello et al. 2008, Pedicelli et al. 2009, Luck \& Lambert 2011, and references therein) and for a few dozen of MC Cepheids. However, the empirical scenario will have a relevant jump thanks to the ongoing massive ground-based spectroscopic surveys at the $8 \mathrm{~m}$ class telescopes (Gaia ESO Survey, Gilmore et al. 2012, Tolstoy et al. 2009)

4. Plain physical arguments indicate that FO Cepheids have the potential to be robust distance 
indicators (Bono et al. 2000). However, we still lack for these variables NIR template light curves. Moreover, current FO absolute calibrations are also hampered by the lack of precise distance determinations based on trigonometric parallaxes for a good sample of Galactic calibrators. These circumstantial evidence limits the precision of $\mathrm{MC}$ distance determinations based on FO Cepheids.

5. Absolute distances based on PW relations including the $\mathrm{H}$ band are characterized by a large spread. The reasons for this behavior are not clear. No doubt that new high-resolution, high signal-tonoise NIR spectra of Galactic Cepheids (Bono et al. 2012) can shed new lights on this open problem.
It is a pleasure to thank an anonymous referee for his/her pertinent suggestions and criticisms that helped us to improve the readability of the paper. We also acknowledge M. Fabrizio for many useful discussions concerning the use of Biweight and data set cleaning. One of us (G.B.) thanks the ESO for support as a science visitor. This work was partially supported by PRIN-INAF 2011 "Tracing the formation and evolution of the Galactic halo with VST" (P.I.: M. Marconi) and by PRINMIUR (2010LY5N2T) "Chemical and dynamical evolution of the Milky Way and Local Group galaxies" (P.I.: F. Matteucci).

\section{REFERENCES}

Alcock, C., Allsman, R. A., Alves, D. R., et al. 2000, ApJ, 542, 281

Benedict, G. F., McArthur, B. E., Feast, M. W., et al. 2007, AJ, 133,1810

Beaulieu, J. P. \& Marquette, J. B. 2000, in ASP Conf. Ser. 203, The Impact of Large-Scale Surveys on Pulsating Star Research, ed. L. Szabados \& D: Kurtz (IAU Colloq. 176; San Francisco,CA: ASP), 139

Bono, G., Caputo, F., Marconi, M., \& Musella, I. 2010, ApJ, 715 277

Bono, G., Caputo, F., Castellani, V., \& Marconi, M. 1999, ApJ, 512,711

Bono, G., Castellani, V., \& Marconi, M. 2000, ApJ, 529, 293

Bono, G., Groenewegen, M. A. T., Marconi, M., \& Caputo, F. 2002, ApJ, 574, L33

Bono, G., \& Marconi, M. 1999, in IAU Symp. 190, New Views of the Magellanic Clouds, ed. Y.-H. Chu, N. Suntzeff, J. Hesser, \& D. Bohlender (Cambridge: Cambridge University Press), 527

Bono,G., Matsunaga, N., Inno, L.,Lagioia, E.P., \& Genovali, K. 2013, in Cosmic-Ray in star-forming environments, ed. D.F. Torres,O. Reimer (Sant Cugat Forum in Astrophysics; Berlin: Springer), in press

Cardelli, J. A., Clayton, G. C., \& Mathis, J. S. 1989, ApJ, 345, 245

Carpenter, J. M. 2001, AJ, 121, 2851

Cioni, M.-R. L., Clementini, G., Girardi, L., et al. 2011, A\&A, 527, A116

Di Criscienzo, M., Marconi, M., Musella, I., Cignoni, M., \& Ripepi, V. 2012, MNRAS, 36, 212

Fabrizio, M., Nonino, M., Bono, G., et al. 2011, PASP, 123, 384

Feast, M. W., \& Catchpole, R. M. 1997, MNRAS, 286, L1

Freedman, W. L., \& Madore, B. F. 2010a, ARA\&A, 48, 673

Freedman, W. L., \& Madore, B. F. 2010b, ApJ, 719, 335

Freedman, W. L., Madore, B. F., Gibson, B. K., et al. 2001, ApJ, 553,47

Freedman, W. L., Madore, B. F., Scowcroft, V., et al. 2012, ApJ, 758,24

Gilmore, G., Randich, S., Asplund, M., et al. 2012, The Messenger, 147, 25

Groenewegen, M. A. T. 2000, A\&A, 363, 901

Groenewegen, M. A. T., \& Salaris, M. 2003, A\&A, 410, 887

Kato, D., Nagashima, C., Nagayama, T., et al. 2007, PASJ, 59, 615

Koen, C., Kanbur, S., \& Ngeow, C. 2007, MNRAS, 380, 1440

Kudritzki, R.-P., \& Urbaneja, M. A. 2012, Ap\&SS, 65, 131

Laney, C. D., \& Stobie, R. S. 1986, MNRAS, 222, 449

Laney, C. D., \& Stobie, R. S. 1992, A\&AS, 93, 93

Laney, C. D., \& Stobie, R. S. 1994, MNRAS, 266, 441

Luck, R. E., \& Lambert, D. L. 2011, AJ, 142, 136

Madore, B. F. 1982, ApJ, 253, 575

Madore, B. F., \& Freedman, W. L. 2009, ApJ, 696, 1498

Majaess, D., Turner, D., \& Gieren, W. 2011, ApJ, 741, L36

Marconi, M., Musella, I., \& Fiorentino, G. 2005, ApJ, 632, 590

Marengo, M., Evans, N. R., Barmby, P., et al. 2010, ApJ, 709, 120
Matsunaga, N., Feast, M. W., \& Menzies, J. W. 2009, MNRAS, 397,933

Matsunaga, N., Feast, M. W., \& Soszyński, I. 2011, MNRAS, 413, 223

McCall, M. L. 2004, AJ, 128, 2144

Meixner, M., Gordon, K. D., Indebetouw, R., et al. 2006, AJ, 132, 2268

Minniti, D., Lucas, P. W., Emerson, J. P., et al. 2010, New Astronomy, 15, 433

Ngeow, C., \& Kanbur, S. M. 2008, Galaxies in the Local Volume, ed. Koribalski, B. S. \& Jerjen, H. (Amsterdarm: Springer), 317 Ngeow, C.-C. 2012, ApJ, 747, 50 (N12)

Ngeow, C.-C., Kanbur, S. M., Neilson, H. R., et al. 2009, ApJ, 693,691

Ngeow, C.-C., Kanbur, S. M., Nikolaev, S., et al. 2005, MNRAS, 363,831

Nikolaev, S., Drake, A. J., Keller, S. C., et al. 2004, ApJ, 601, 260

Pedicelli, S., Bono, G., Lemasle, B., et al. 2009, A\&A, 504, 81

Persson, S. E., Madore, B. F., Krzemiński, W., et al. 2004, AJ, 128, 2239 (P04)

Pietrzyński, G., Thompson, I. B., Gieren, W., et al. 2010, Nature, 468,542

Pietrzyński, G., Thompson, I. B., Graczyk, D., et al. 2011, ApJ, 742, L20

Riess, A. G., Macri, L., Casertano, S., et al. 2011, ApJ, 730, 119

Ripepi, V., Moretti, M. I., Marconi, M., et al. 2012, MNRAS, 424, 1807 (R12)

Romaniello, M., Primas, F., Mottini, M., et al. 2008, A\&A, 488, 731

Sandage, A., Tammann, G. A., \& Reindl, B. 2004, A\&A, 424, 43

Sandage, A., Tammann, G. A., \& Reindl, B. 2009, A\&A, 493, 471

Sasselov, D. D., Beaulieu, J. P., Renault, C., et al. 1997, A\&A, 324,471

Scowcroft, V., Freedman, W. L., Madore, B. F., et al. 2011, ApJ, 743,76

Skrutskie, M. F., Cutri, R. M., Stiening, R., et al. 2006, AJ, 131, 1163

Soszyński, I., Gieren, W., \& Pietrzyński, G. 2005, PASP, 117, 823

Soszyńnski, I., Poleski, R., Udalski, A., et al. 2008, AcA, 58, 163

Soszyński, I., Poleski, R., Udalski, A., et al. 2010, AcA, 60, 17

Soszyński, I., Udalski, A., Poleski, R., et al. 2012, AcA, 62, 219

Storm, J., Gieren, W., Fouqué, P., et al. 2011, A\&A, 534, A94 (S11a)

Storm, J., Gieren, W., Fouqué, P., et al. 2011, A\&A, 534, A95 (S11b)

Tolstoy, E., Hill, V., \& Tosi, M. 2009, ARA\&A, 47, 371

Udalski, A., Szymanski, M., Kubiak, M., et al. 1999, AcA, 49, 201

van den Bergh, S. 2000, The galaxies of the Local Group, (Cambridge, Cambridge University Press), 92

van Leeuwen, F., Feast, M. W., Whitelock, P. A., \& Laney, C. D. 2007, MNRAS, 379, 723

Welch, D. L. , \& MACHO Collaboration 1999, in IAU Symp. 190, New Views of the Magellanic Clouds, ed. Y.-H. Chu, N. Suntzeff, J. Hesser, \& D. Bohlender (Cambridge: Cambridge Univ. Press), 513 
Welch, D. L., McLaren, R. A., Madore, B. F., \& McAlary, C. W. 1987, ApJ, 321, 162 
TABLE 1

NIR and Optical-NIR PW RELATions For LMC and SMC Cepheids.

\begin{tabular}{|c|c|c|c|c|c|c|}
\hline $\mathrm{W}\left(\lambda_{2}, \lambda_{1}\right)^{\mathrm{a}}$ & Mode & $\mathrm{a}$ & $\mathrm{b}$ & $\sigma^{\mathrm{b}}$ & $\mu_{\pi}$ & $\mu_{\text {theo }}$ \\
\hline \multicolumn{7}{|c|}{ LMC } \\
\hline $\mathrm{W}\left(J, K_{\mathrm{S}}\right)$ & FU (1708) & $15.876 \pm 0.005$ & $-3.365 \pm 0.008$ & 0.08 & $18.44 \pm 0.05^{\mathrm{c}}$ & $18.53 \pm 0.07^{\mathrm{d}}$ \\
\hline $\mathrm{W}(J, H)$ & FU (1701) & $15.630 \pm 0.006$ & $-3.373 \pm 0.008$ & 0.08 & $18.30 \pm 0.05^{\mathrm{c}}$ & $18.65 \pm 0.04^{\mathrm{d}}$ \\
\hline $\mathrm{W}\left(H, K_{\mathrm{S}}\right)$ & FU (1709) & $16.058 \pm 0.006$ & $-3.360 \pm 0.010$ & 0.10 & $18.54 \pm 0.05^{\mathrm{c}}$ & $18.46 \pm 0.12^{\mathrm{d}}$ \\
\hline $\mathrm{W}\left(V, K_{\mathrm{S}}\right)$ & FU (1737) & $15.901 \pm 0.005$ & $-3.326 \pm 0.008$ & 0.07 & $18.46 \pm 0.05^{\mathrm{c}}$ & $18.51 \pm 0.08^{\mathrm{d}}$ \\
\hline $\mathrm{W}(V, H)$ & FU (1730) & $15.816 \pm 0.005$ & $-3.315 \pm 0.008$ & 0.07 & $18.40 \pm 0.05^{\mathrm{c}}$ & $18.56 \pm 0.06^{\mathrm{d}}$ \\
\hline $\mathrm{W}(V, J)$ & FU (1732) & $15.978 \pm 0.006$ & $-3.272 \pm 0.009$ & 0.08 & $18.49 \pm 0.05^{\mathrm{c}}$ & $18.47 \pm 0.12^{\mathrm{d}}$ \\
\hline $\mathrm{W}\left(I, K_{\mathrm{S}}\right)$ & FU (1737) & $15.902 \pm 0.005$ & $-3.325 \pm 0.008$ & 0.07 & $18.46 \pm 0.05^{\mathrm{c}}$ & $18.52 \pm 0.08^{\mathrm{d}}$ \\
\hline $\mathrm{W}(I, H)$ & FU (1734) & $15.801 \pm 0.005$ & $-3.317 \pm 0.008$ & 0.08 & $18.39 \pm 0.05^{\mathrm{c}}$ & $18.55 \pm 0.06^{\mathrm{d}}$ \\
\hline $\mathrm{W}(I, J)$ & FU (1735) & $16.002 \pm 0.007$ & $-3.243 \pm 0.011$ & 0.10 & $18.50 \pm 0.05^{\mathrm{c}}$ & $18.46 \pm 0.12^{\mathrm{d}}$ \\
\hline $\mathrm{W}(V, I)$ & FU (1700) & $15.899 \pm 0.005$ & $-3.327 \pm 0.008$ & 0.07 & $18.47 \pm 0.05^{\mathrm{c}}$ & $18.53 \pm 0.13^{\mathrm{d}}$ \\
\hline MEAN (FU) & & & & & $18.45 \pm 0.02^{\mathrm{e}}$ & $18.56 \pm 0.02^{\mathrm{e}}$ \\
\hline $\mathrm{W}\left(J, K_{\mathrm{S}}\right)$ & FO (1057) & $15.370 \pm 0.005$ & $-3.471 \pm 0.013$ & 0.08 & $18.60 \pm 0.08^{\mathrm{f}}$ & $18.52 \pm 0.06^{\mathrm{g}}$ \\
\hline $\mathrm{W}(J, H)$ & FO (1064) & $15.207 \pm 0.005$ & $-3.507 \pm 0.015$ & 0.09 & $18.60 \pm 0.08^{\mathrm{f}}$ & $18.56 \pm 0.06^{\mathrm{g}}$ \\
\hline $\mathrm{W}\left(H, K_{\mathrm{S}}\right)$ & FO (1063) & $15.483 \pm 0.007$ & $-3.425 \pm 0.017$ & 0.10 & $18.59 \pm 0.08^{\mathrm{f}}$ & $18.49 \pm 0.07^{\mathrm{g}}$ \\
\hline $\mathrm{W}\left(V, K_{\mathrm{S}}\right)$ & FO (1061) & $15.410 \pm 0.005$ & $-3.456 \pm 0.013$ & 0.07 & $18.61 \pm 0.08^{\mathrm{f}}$ & $18.51 \pm 0.06^{\mathrm{g}}$ \\
\hline $\mathrm{W}(V, H)$ & FO (1071) & $15.357 \pm 0.004$ & $-3.485 \pm 0.011$ & 0.08 & $18.61 \pm 0.08^{\mathrm{f}}$ & $18.52 \pm 0.06^{\mathrm{g}}$ \\
\hline $\mathrm{W}(V, J)$ & FO (1086) & $15.475 \pm 0.005$ & $-3.434 \pm 0.014$ & 0.10 & $18.62 \pm 0.08^{\mathrm{f}}$ & $18.48 \pm 0.06^{\mathrm{g}}$ \\
\hline $\mathrm{W}\left(I, K_{\mathrm{S}}\right)$ & FO (1059) & $15.402 \pm 0.005$ & $-3.448 \pm 0.013$ & 0.08 & $18.61 \pm 0.08^{\mathrm{f}}$ & $18.50 \pm 0.06^{\mathrm{g}}$ \\
\hline $\mathrm{W}(I, H)$ & FO (1072) & $15.351 \pm 0.004$ & $-3.489 \pm 0.012$ & 0.08 & $18.62 \pm 0.08^{\mathrm{f}}$ & $18.51 \pm 0.06^{\mathrm{g}}$ \\
\hline $\mathrm{W}(I, J)$ & FO (1100) & $15.499 \pm 0.006$ & $-3.423 \pm 0.020$ & 0.13 & $18.66 \pm 0.08^{\mathrm{f}}$ & $18.45 \pm 0.06^{\mathrm{g}}$ \\
\hline $\mathrm{W}(V, I)$ & FO (1081) & $15.399 \pm 0.003$ & $-3.460 \pm 0.009$ & 0.07 & $18.52 \pm 0.06^{\mathrm{f}}$ & $18.56 \pm 0.06^{\mathrm{g}}$ \\
\hline MEAI & & & & & $18.60 \pm 0.03^{\mathrm{e}}$ & $18.51 \pm 0.02^{\mathrm{e}}$ \\
\hline \multicolumn{7}{|c|}{$\mathrm{SMC}$} \\
\hline $\mathrm{W}\left(J, K_{\mathrm{S}}\right)$ & FU (2448) & $16.457 \pm 0.006$ & $-3.480 \pm 0.011$ & 0.16 & $18.92 \pm 0.05^{\mathrm{c}}$ & $19.01 \pm 0.10^{\mathrm{d}}$ \\
\hline $\mathrm{W}(J, H)$ & FU (2448) & $16.217 \pm 0.006$ & $-3.542 \pm 0.011$ & 0.17 & $18.74 \pm 0.05^{\mathrm{c}}$ & $19.02 \pm 0.07^{\mathrm{d}}$ \\
\hline $\mathrm{W}\left(H, K_{\mathrm{S}}\right)$ & FU (2448) & $16.638 \pm 0.006$ & $-3.445 \pm 0.011$ & 0.19 & $19.05 \pm 0.05^{\mathrm{c}}$ & $19.01 \pm 0.14^{\mathrm{d}}$ \\
\hline $\mathrm{W}\left(V, K_{\mathrm{S}}\right)$ & FU (2295) & $16.507 \pm 0.005$ & $-3.461 \pm 0.011$ & 0.15 & $18.95 \pm 0.05^{\mathrm{c}}$ & $19.00 \pm 0.11^{\mathrm{d}}$ \\
\hline $\mathrm{W}(V, H)$ & FU (2285) & $16.426 \pm 0.005$ & $-3.475 \pm 0.010$ & 0.15 & $18.88 \pm 0.05^{\mathrm{c}}$ & $19.00 \pm 0.10^{\mathrm{d}}$ \\
\hline $\mathrm{W}(V, J)$ & FU (2286) & $16.614 \pm 0.005$ & $-3.427 \pm 0.011$ & 0.16 & $19.00 \pm 0.05^{\mathrm{c}}$ & $18.98 \pm 0.14^{\mathrm{d}}$ \\
\hline $\mathrm{W}\left(I, K_{\mathrm{S}}\right)$ & FU (2294) & $16.511 \pm 0.005$ & $-3.464 \pm 0.011$ & 0.16 & $18.95 \pm 0.05^{\mathrm{c}}$ & $19.00 \pm 0.10^{\mathrm{d}}$ \\
\hline $\mathrm{W}(I, H)$ & FU (2202) & $16.417 \pm 0.005$ & $-3.480 \pm 0.011$ & 0.15 & $18.87 \pm 0.05^{\mathrm{c}}$ & $19.00 \pm 0.10^{\mathrm{d}}$ \\
\hline $\mathrm{W}(I, J)$ & FU (2279) & $16.662 \pm 0.006$ & $-3.424 \pm 0.013$ & 0.18 & $19.01 \pm 0.05^{\mathrm{c}}$ & $18.92 \pm 0.14^{\mathrm{d}}$ \\
\hline $\mathrm{W}(V, I)$ & $\mathrm{FU}(2260)$ & $16.482 \pm 0.005$ & $-3.449 \pm 0.010$ & 0.13 & $18.95 \pm 0.05^{\mathrm{c}}$ & $19.03 \pm 0.12^{\mathrm{d}}$ \\
\hline MEAN & & & & & $18.93 \pm 0.02^{\mathrm{e}}$ & $18.99 \pm 0.03^{\mathrm{e}}$ \\
\hline $\mathrm{W}\left(J, K_{\mathrm{S}}\right)$ & FO (1461) & $15.947 \pm$ & $-3.651 \pm 0.022$ & 0.16 & $19.06 \pm 0.08^{\mathrm{f}}$ & $19.02 \pm 0.04^{\mathrm{g}}$ \\
\hline $\mathrm{W}(J, H)$ & FO (1473) & $15.778 \pm 0.006$ & $-3.722 \pm 0.023$ & 0.17 & $19.17 \pm 0.08^{\mathrm{f}}$ & $19.05 \pm 0.03^{\mathrm{g}}$ \\
\hline $\mathrm{W}\left(H, K_{\mathrm{S}}\right)$ & FO (1456) & $16.069 \pm 0.007$ & $-3.579 \pm 0.027$ & 0.19 & $19.00 \pm 0.08^{\mathrm{f}}$ & $19.01 \pm 0.04^{\mathrm{g}}$ \\
\hline $\mathrm{W}\left(V, K_{\mathrm{S}}\right)$ & FO (1472) & $15.992 \pm 0.005$ & $-3.624 \pm 0.021$ & 0.16 & $19.09 \pm 0.08^{\mathrm{f}}$ & $19.02 \pm 0.04^{\mathrm{g}}$ \\
\hline $\mathrm{W}(V, H)$ & FO (1482) & $15.937 \pm 0.005$ & $-3.660 \pm 0.020$ & 0.15 & $19.16 \pm 0.08^{\mathrm{f}}$ & $19.03 \pm 0.05^{\mathrm{g}}$ \\
\hline $\mathrm{W}(V, J)$ & FO (1494) & $16.074 \pm 0.006$ & $-3.578 \pm 0.023$ & 0.18 & $19.17 \pm 0.08^{\mathrm{f}}$ & $19.02 \pm 0.04^{\mathrm{g}}$ \\
\hline $\mathrm{W}\left(I, K_{\mathrm{S}}\right)$ & FO (1471) & $15.990 \pm 0.005$ & $-3.630 \pm 0.020$ & 0.16 & $19.09 \pm 0.08^{\mathrm{f}}$ & $19.02 \pm 0.05^{\mathrm{g}}$ \\
\hline $\mathrm{W}(I, H)$ & FO (1477) & $15.932 \pm 0.005$ & $-3.667 \pm 0.020$ & 0.16 & $19.17 \pm 0.08^{\mathrm{f}}$ & $19.02 \pm 0.04^{\mathrm{g}}$ \\
\hline $\mathrm{W}(I, J)$ & FO (1499) & $16.113 \pm 0.007$ & $-3.595 \pm 0.027$ & 0.20 & $19.17 \pm 0.08^{\mathrm{f}}$ & $18.00 \pm 0.05^{\mathrm{g}}$ \\
\hline $\mathrm{W}(V, I)$ & FO (1465) & $15.958 \pm 0.005$ & $-3.599 \pm 0.019$ & 0.14 & $19.12 \pm 0.06^{\mathrm{f}}$ & $19.05 \pm 0.03^{\mathrm{g}}$ \\
\hline MEAN (FO) & & & & & $19.12 \pm 0.03^{\mathrm{e}}$ & $19.02 \pm 0.02^{\mathrm{e}}$ \\
\hline
\end{tabular}

a The color coefficients of the adopted PW relations are the following: $\frac{A_{K}}{E\left(J-K_{\mathrm{S}}\right)}=0.69 ; \frac{A_{H}}{E(J-H)}=1.63$; $\frac{A_{K}}{E\left(H-K_{\mathrm{S}}\right)}=1.92 ; \frac{A_{K}}{E\left(V-K_{\mathrm{S}}\right)}=0.13 ; \frac{A_{H}}{E(V-H)}=0.22 ; \frac{A_{J}}{E(V-J)}=0.41 ; \frac{A_{K}}{E\left(I-K_{\mathrm{S}}\right)}=0.24 ; \frac{A_{H}}{E(I-H)}=0.42 ; \frac{A_{J}}{E(I-J)}=0.92 ;$ $\frac{A_{I}}{E(I-V)}=1.55$

b Dispersion of the linear fit (mag)

c Distance modulus based on the zero-point calibration from Benedict et al. (2007).

d Distance modulus based on the zero-point calibration obtained by the predicted FU PW relations for Magellanic Cepheids provided by Marconi et al. 2005. The associated error is the dispersion of the theoretical PW relation.

e Weighted distance modulus estimated using the distance moduli of individual PW relations.

${ }^{\mathrm{f}}$ Distance modulus obtained using Polaris for the zero-point calibration (van Leeuwen et al. 2007).

g Distance modulus based on the zero-point calibration obtained by the predicted FO PW relations for Magellanic Cepheids provided by Marconi et al. (2005). The associated error is the dispersion of the theoretical PW relation. 
TABLE 2

Difference in the SLOPES OF THE PW RELATIONS FOR LMC AND SMC CEPHEIDS.

\begin{tabular}{|c|c|c|c|}
\hline $\mathrm{W}\left(\lambda_{2}, \lambda_{1}\right)$ & Mode & $\Delta \mathrm{b}_{L M C-S M C^{\mathrm{a}}}$ & $\sigma_{t o t} \mathrm{~b}$ \\
\hline $\begin{array}{l}\mathrm{W}\left(J, K_{\mathrm{S}}\right) \\
\mathrm{W}(J, H) \\
\mathrm{W}\left(H, K_{\mathrm{S}}\right) \\
\mathrm{W}\left(V, K_{\mathrm{S}}\right) \\
\mathrm{W}(V, H) \\
\mathrm{W}(V, J) \\
\mathrm{W}\left(I, K_{\mathrm{S}}\right) \\
\mathrm{W}(I, H) \\
\mathrm{W}(I, J) \\
\mathrm{W}(V, I)\end{array}$ & $\begin{array}{l}\text { FU } \\
\text { FU } \\
\text { FU } \\
\text { FU } \\
\text { FU } \\
\text { FU } \\
\text { FU } \\
\text { FU } \\
\text { FU } \\
\text { FU }\end{array}$ & $\begin{array}{l}0.115 \pm 0.014 \\
0.169 \pm 0.014 \\
0.120 \pm 0.015 \\
0.135 \pm 0.014 \\
0.160 \pm 0.013 \\
0.155 \pm 0.014 \\
0.139 \pm 0.014 \\
0.163 \pm 0.014 \\
0.181 \pm 0.017 \\
0.122 \pm 0.014\end{array}$ & $\begin{array}{l}0.18 \\
0.19 \\
0.21 \\
0.17 \\
0.17 \\
0.18 \\
0.17 \\
0.18 \\
0.21 \\
0.15\end{array}$ \\
\hline $\begin{array}{l}\mathrm{W}\left(J, K_{\mathrm{S}}\right) \\
\mathrm{W}(J, H) \\
\mathrm{W}\left(H, K_{\mathrm{S}}\right) \\
\mathrm{W}\left(V, K_{\mathrm{S}}\right) \\
\mathrm{W}(V, H) \\
\mathrm{W}(V, J) \\
\mathrm{W}\left(I, K_{\mathrm{S}}\right) \\
\mathrm{W}(I, H) \\
\mathrm{W}(I, J) \\
\mathrm{W}(V, I)\end{array}$ & $\begin{array}{l}\text { FO } \\
\text { FO } \\
\text { FO } \\
\text { FO } \\
\text { FO } \\
\text { FO } \\
\text { FO } \\
\text { FO } \\
\text { FO } \\
\text { FO }\end{array}$ & $\begin{array}{l}0.180 \pm 0.026 \\
0.215 \pm 0.027 \\
0.154 \pm 0.032 \\
0.172 \pm 0.024 \\
0.175 \pm 0.023 \\
0.143 \pm 0.027 \\
0.182 \pm 0.024 \\
0.178 \pm 0.023 \\
0.172 \pm 0.034 \\
0.139 \pm 0.021\end{array}$ & $\begin{array}{l}0.18 \\
0.19 \\
0.21 \\
0.18 \\
0.17 \\
0.21 \\
0.18 \\
0.18 \\
0.23 \\
0.16\end{array}$ \\
\hline $\begin{array}{l}\text { a The error o } \\
\text { counting for } \mathrm{t} \\
\text { relations. } \\
\mathrm{b} \text { Total dispe } \\
\text { where } \sigma_{L M C} \\
\text { persions of th } \\
\text { Cepheids, res }\end{array}$ & on, & $\sigma_{t o t}=\sqrt{\sigma_{L M C}^{2}}$ & $\begin{array}{l}\text { oy ac- } \\
\text { f PW } \\
\frac{2}{S M C}, \\
1 \text { dis- } \\
\text { SMC }\end{array}$ \\
\hline
\end{tabular}


TABLE 3

NIR AND OPTICAL-NIR PW RELATIONS FOR SMC CEPHEIDS COMPUTED BY ASSUMING DIFFERENT BREAK POINTS IN PERIOD.

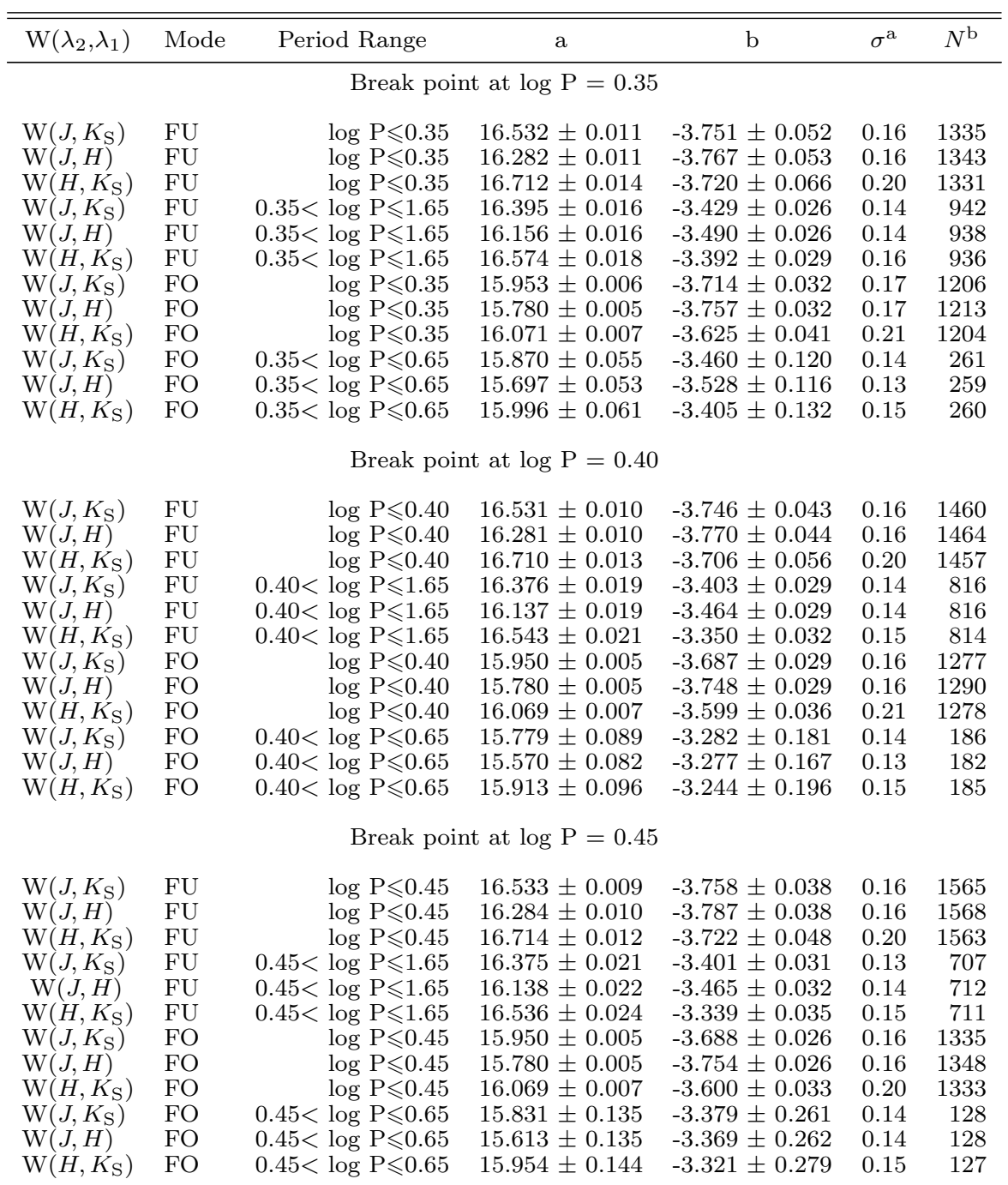

${ }^{a}$ Dispersion of the linear fit (mag).

b Number of Cepheids adopted in the fit.

TABLE 4

NIR PW RELATIONS FOR LMC CEPHEIDS COMPUTED BY ASSUMING A BREAK IN PERIOD AT $\log \mathrm{P}=1$

\begin{tabular}{|c|c|c|c|c|c|c|}
\hline $\mathrm{W}\left(\lambda_{2}, \lambda_{1}\right)$ & Mode & Period Range & $\mathrm{a}$ & $\mathrm{b}$ & $\sigma^{\mathrm{a}}$ & $N^{\mathrm{b}}$ \\
\hline $\mathrm{W}\left(J, K_{\mathrm{S}}\right)$ & $\mathrm{FU}$ & $\log \mathrm{P} \leqslant 1.0$ & $15.884 \pm 0.007$ & $-3.380 \pm 0.011$ & 0.07 & 1674 \\
\hline $\mathrm{W}(J, H)$ & FU & $\log \mathrm{P} \leqslant 1.0$ & $15.676 \pm 0.007$ & $-3.457 \pm 0.012$ & 0.08 & 1675 \\
\hline $\mathrm{W}\left(H, K_{\mathrm{S}}\right)$ & FU & $\log \mathrm{P} \leqslant 1.0$ & $16.039 \pm 0.008$ & $-3.324 \pm 0.014$ & 0.10 & 1684 \\
\hline $\mathrm{W}\left(J, K_{\mathrm{S}}\right)$ & FU & $1.0<\log \mathrm{P} \leqslant 1.65$ & $15.950 \pm 0.071$ & $-3.413 \pm 0.056$ & 0.08 & 69 \\
\hline $\mathrm{W}(J, H)$ & FU & $1.0<\log \mathrm{P} \leqslant 1.65$ & $15.778 \pm 0.084$ & $-3.419 \pm 0.067$ & 0.10 & 68 \\
\hline $\mathrm{W}\left(H, K_{\mathrm{S}}\right)$ & FU & $1.0<\log \mathrm{P} \leqslant 1.65$ & $16.107 \pm 0.075$ & $-3.437 \pm 0.060$ & 0.09 & 69 \\
\hline
\end{tabular}

${ }^{\mathrm{a}}$ Dispersion of the linear fit (mag).

b Number of Cepheids adopted in the fit. 
TABLE 5

Difference in Distance modulus Between LMC and SMC.

\begin{tabular}{|c|c|c|c|c|c|c|}
\hline $\mathrm{W}\left(\lambda_{2}, \lambda_{1}\right)$ & Mode & $\Delta \mu_{\text {short }}$ & $\Delta \mu_{\text {long }}$ & Break & $\mathrm{x}_{s}{ }^{\mathrm{a}}$ & $\mathrm{x}_{l}^{\mathrm{a}}$ \\
\hline $\mathrm{W}\left(J, K_{\mathrm{S}}\right)$ & $\mathrm{FU}$ & $0.55 \pm 0.06^{\mathrm{b}}$ & $0.47 \pm 0.06^{\mathrm{b}}$ & & 0.3 & 1.0 \\
\hline & $\mathrm{FU}$ & $0.53 \pm 0.11$ & $0.48 \pm 0.13$ & 0.35 & 0.3 & 1.0 \\
\hline$"$ & $\mathrm{FU}$ & $0.54 \pm 0.12$ & $0.44 \pm 0.13$ & 0.40 & 0.3 & 1.0 \\
\hline$"$ & FU & $0.54 \pm 0.09$ & $0.48 \pm 0.13$ & 0.45 & 0.3 & 1.0 \\
\hline $\mathrm{W}(J, H)$ & $\mathrm{FU}$ & $0.55 \pm 0.05^{\mathrm{b}}$ & $0.42 \pm 0.06^{\mathrm{b}}$ & & 0.3 & 1.0 \\
\hline & FU & $0.51 \pm 0.11$ & $0.31 \pm 0.16$ & 0.35 & 0.3 & 1.0 \\
\hline$"$ & FU & $0.51 \pm 0.10$ & $0.31 \pm 0.16$ & 0.40 & 0.3 & 1.0 \\
\hline$"$ & FU & $0.50 \pm 0.10$ & $0.31 \pm 0.16$ & 0.45 & 0.3 & 1.0 \\
\hline $\mathrm{W}\left(H, K_{\mathrm{S}}\right)$ & $\mathrm{FU}$ & $0.56 \pm 0.07^{\mathrm{b}}$ & $0.50 \pm 0.08^{\mathrm{b}}$ & & 0.3 & 1.0 \\
\hline , & $\mathrm{FU}$ & $0.56 \pm 0.12$ & $0.52 \pm 0.16$ & 0.35 & 0.3 & 1.0 \\
\hline$"$ & FU & $0.56 \pm 0.14$ & $0.51 \pm 0.15$ & 0.40 & 0.3 & 1.0 \\
\hline$"$ & FU & $0.56 \pm 0.13$ & $0.52 \pm 0.17$ & 0.45 & 0.3 & 1.0 \\
\hline
\end{tabular}

Note. - The errors on the relative distances were estimated by accounting for the uncertainties both in the coefficients and in the dispersion of the individual PW relations.

a $\Delta \mu_{\text {short }}=a 1_{s}+b 1_{s} * x_{s}-\left(a 2_{s}+b 2 * x_{s}\right)$ and $\Delta \mu_{l o n g}=a 1_{l}+b 1_{l} * x_{l}-$ $\left(a 2_{l}+b 2 * x_{l}\right)$, where the index 1 refers to the SMC linear regressions, while the index 2 refers to the LMC linear regressions.

$\mathrm{b}$ Relative distance moduli estimated using the PW relations on the entire range of periods. $\Delta \mu_{\text {short }}$ here is the relative distance moduli obtained at $\log P=x_{s}$, while $\Delta \mu_{\text {long }}$ is obtained at $\log P=x_{l}$

TABLE 6

Optical AND NIR PW Relations aVAilable in the Literature.

\begin{tabular}{lcccccc}
\hline \hline $\mathrm{W}\left(\lambda_{2}, \lambda_{1}\right)$ & Mode & $\mathrm{a}$ & \multicolumn{1}{c}{$\mathrm{b}$} & $\sigma$ & galaxy & Reference \\
\hline $\mathrm{W}\left(J, K_{\mathrm{S}}\right)$ & $\mathrm{FU}(229)$ & $-2.65 \pm 0.02$ & $-3.34 \pm 0.03$ & 0.10 & $\mathrm{MW}$ & $\mathrm{N} 12$ \\
$\mathrm{~W}\left(J, K_{\mathrm{S}}\right)$ & $\mathrm{FU}(70)$ & $-2.52 \pm 0.12$ & $-3.44 \pm 0.09$ & 0.23 & $\mathrm{MW}$ & $\mathrm{S} 11 \mathrm{a}$ \\
$\mathrm{W}(V, I)$ & $\mathrm{FU}(70)$ & $-2.70 \pm 0.15$ & $-3.26 \pm 0.11$ & 0.26 & $\mathrm{MW}$ & $\mathrm{S} 11 \mathrm{a}$ \\
$\mathrm{W}(V, I)$ & $\mathrm{FU}(10)$ & $-2.48 \pm 0.15$ & $-3.37 \pm 0.12$ & 0.11 & $\mathrm{MW}$ & $\mathrm{B} 07$ \\
$\mathrm{~W}\left(V, K_{\mathrm{S}}\right)$ & $\mathrm{FU}(10)$ & $-2.60 \pm 0.07$ & $-3.325 \pm 0.014$ & 0.08 & LMC & R12 \\
& & & & & \\
\hline
\end{tabular}




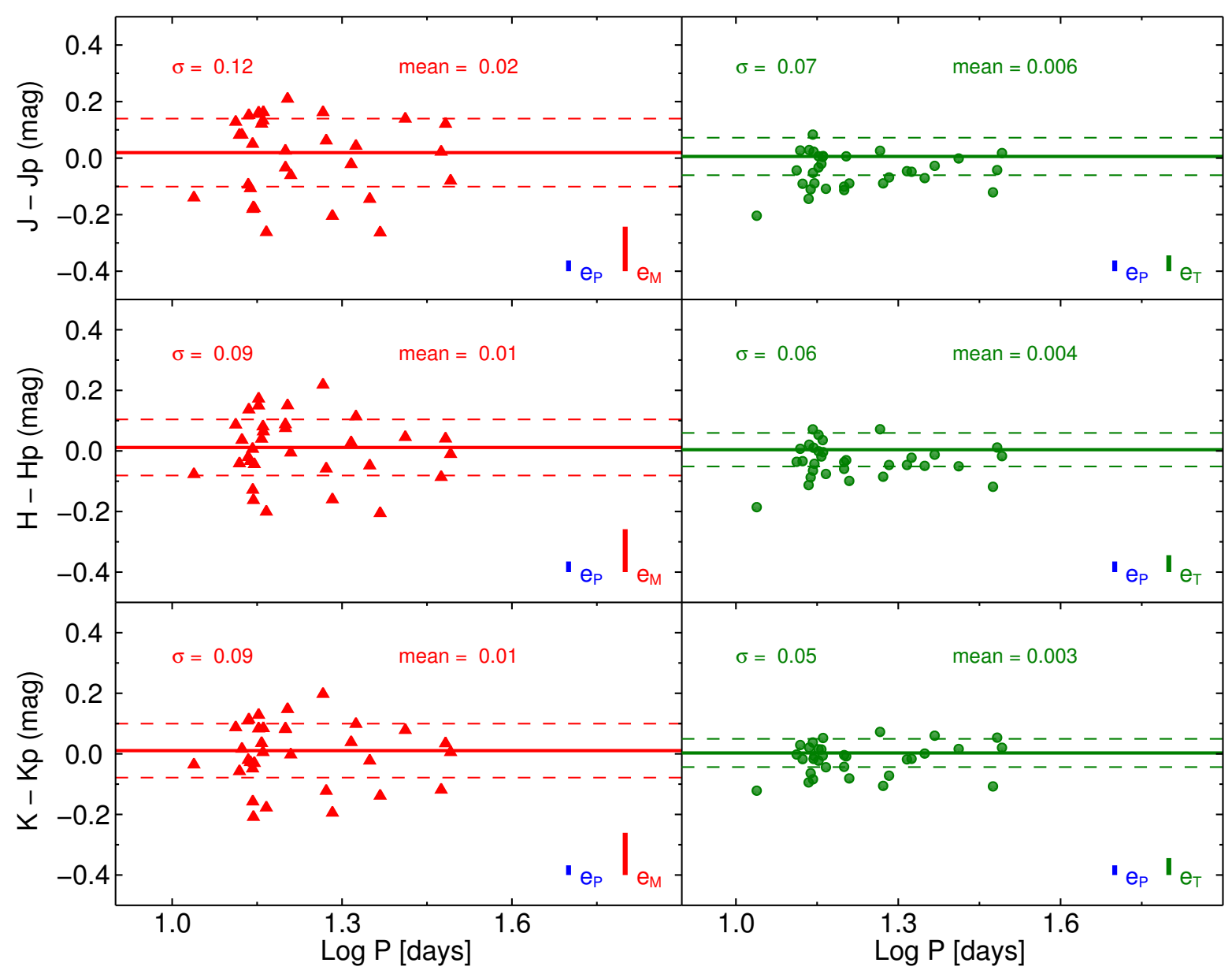

Fig. 1. - Left: difference between the $J$ (top), $H$ (middle) and $K_{\mathrm{S}}$ (bottom) Cepheid single epoch magnitudes and the true $J p, H p, K p_{\mathrm{S}}$ mean magnitudes from P04. For each band is plotted the mean difference (solid red line) and the dispersion ( $\sigma$, dashed red line). The blue and the red bars in the right corner show the typical photometric error associated to the true mean magnitudes $\left(e_{P}\right)$ and the error of IRSF magnitudes ( $e_{M}$, photometric error plus the semi-amplitude). Right: Same as the left, but difference is between the $J$ (top), $H$ (middle) and $K_{\mathrm{S}}$ (bottom) mean magnitudes obtained by applying the template to single epoch magnitudes and the true $J p, H p, K p_{\mathrm{S}}$ mean magnitudes from P04. The solid and dashed green lines have the same meaning, while the green bar shows the total error $\left(e_{T}\right.$, photometric error plus error of the template light curve). 


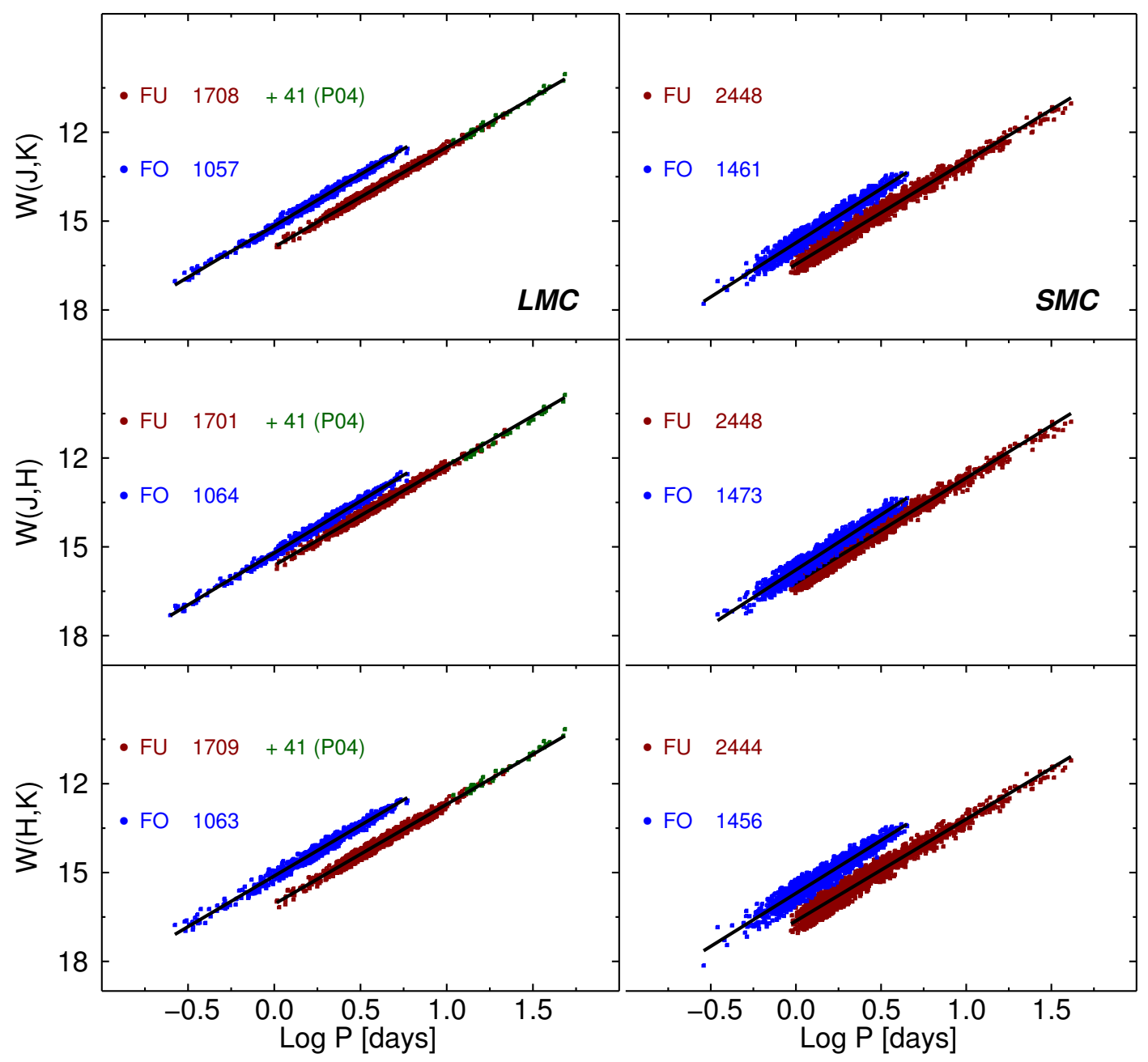

FIG. 2.- NIR Period-Wesenheit relations for LMC (left) and SMC (right) Cepheids. Red dots display IRSF mean magnitudes for FU pulsators, while green dots are the mean magnitude for 41 Cepheids by P04. Blue dots show the FO pulsators. The solid lines show the linear fits. 


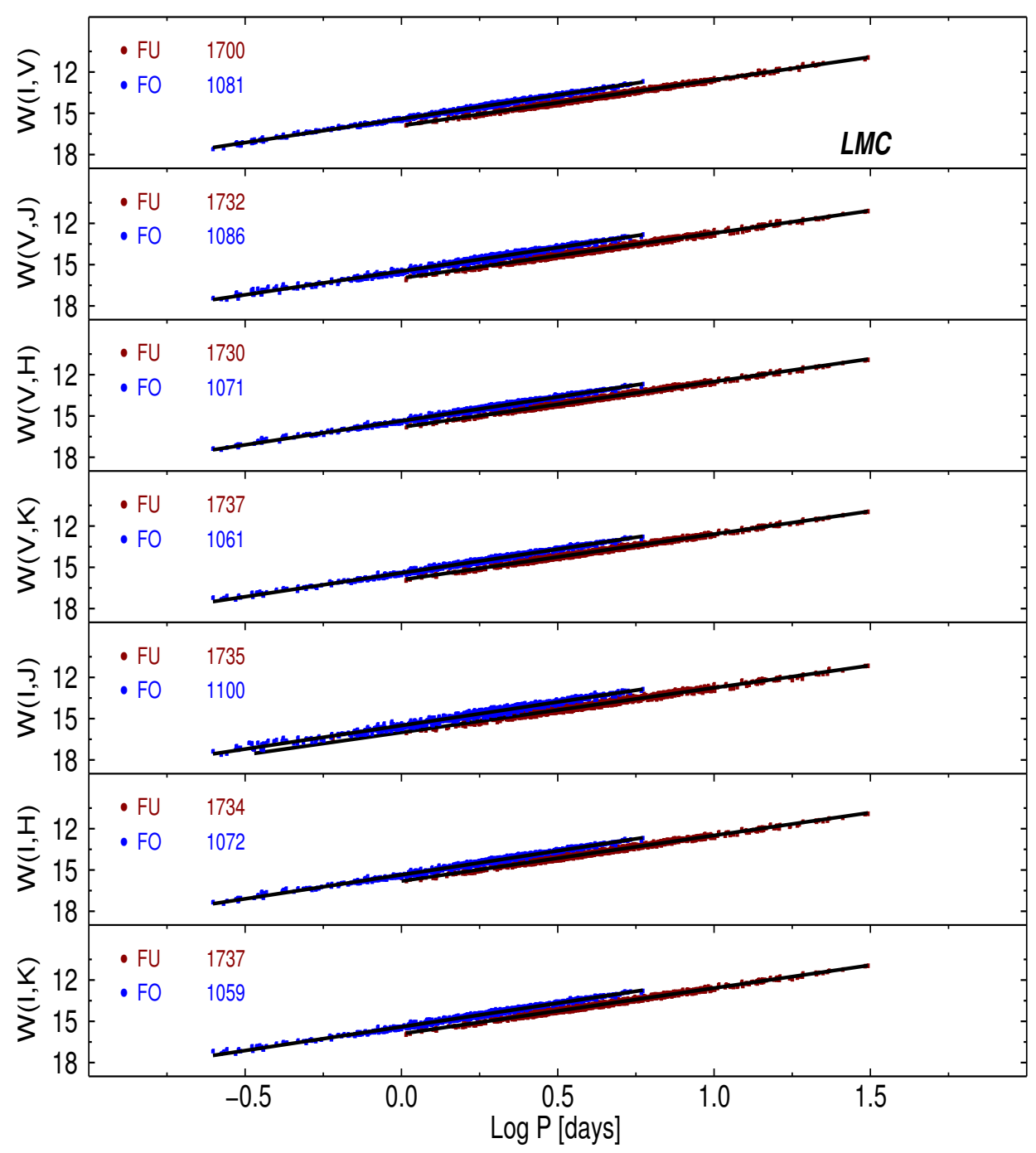

FIG. 3.- Optical-NIR Period-Wesenheit relations for LMC Cepheids. Symbols and lines are the same as Figure 2. 


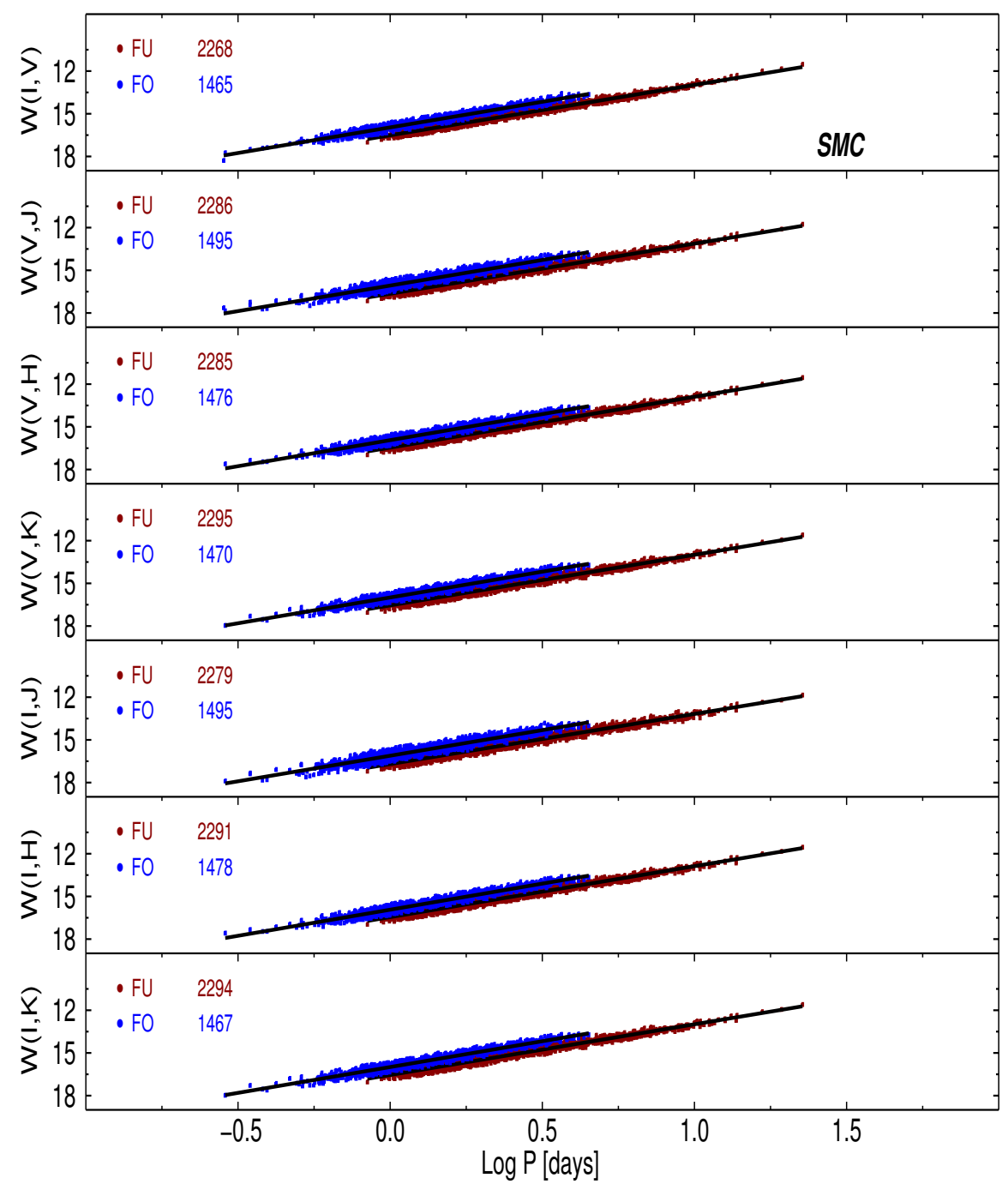

FIG. 4.- Same as Figure 3, but for SMC Cepheids. 

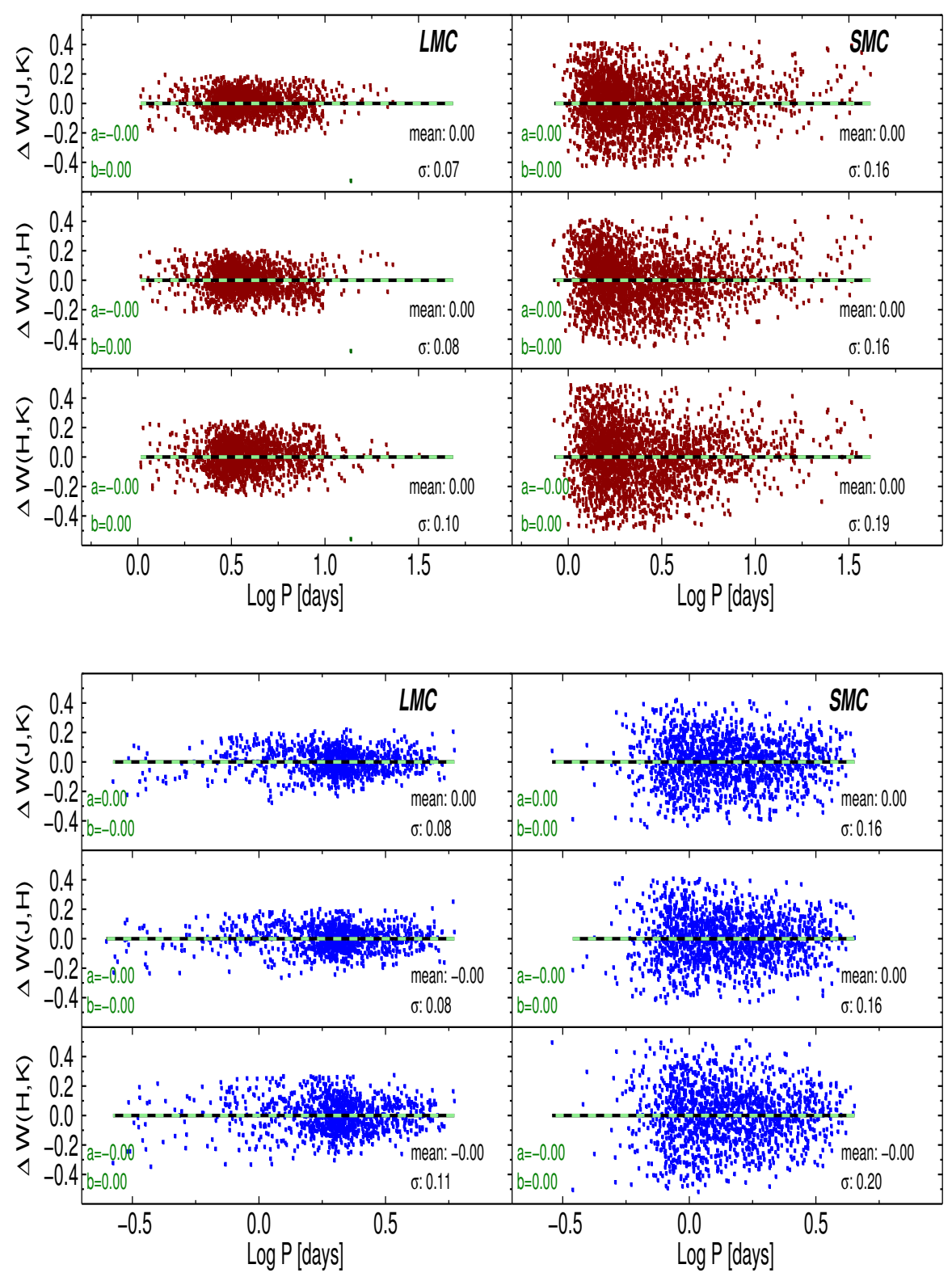

FIG. 5.- Top - Difference between individual NIR Wesenheit mean magnitudes of LMC (left) and SMC (right) Cepheids and the PW relations. The linear fit of the residuals is also overplotted (dashed green line). The weighted means and the intrinsic dispersions are also labeled. Bottom - Same as the top but for FO Cepheids. 


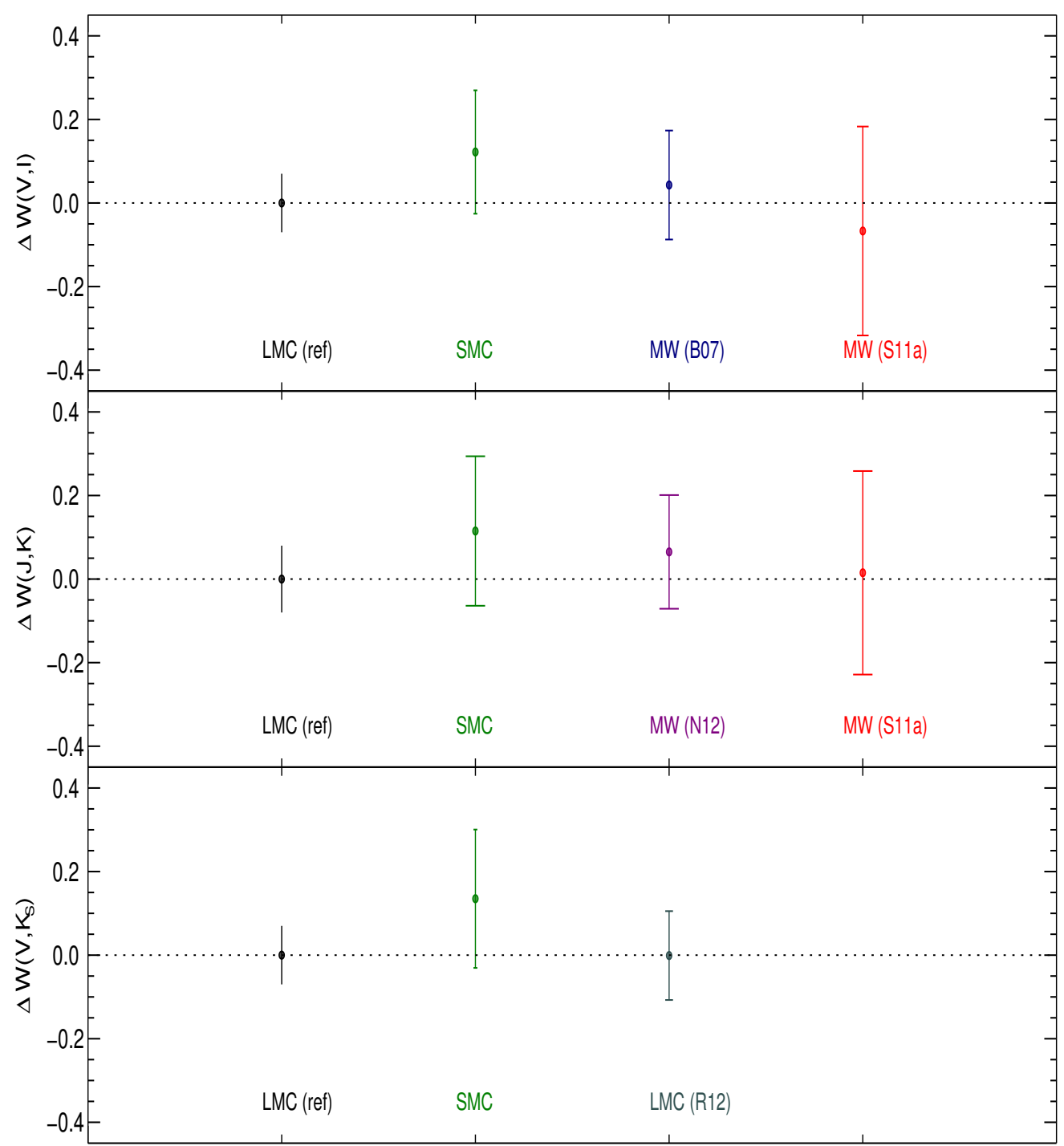

FIG. 6. - Top - Difference in the slope between the PW $(V, I)$ relation for the LMC (black), SMC (green) and similar slopes for Galactic Cepheids provided by B07 (blue) and by S11a (red). The vertical error bars display the dispersion of the different PW relations. Middle - Same as the top, but for the PW $\left(J, K_{\mathrm{S}}\right)$ relations. The slopes for Galactic Cepheids were provided by N12 (purple) and by S11a (red). Bottom - Same as the top, but for the PW $\left(V, K_{\mathrm{S}}\right)$ relations. The slope for LMC Cepheids was provided by R12 (grey). 